\title{
Plasma neutrophil gelatinase-associated lipocalin (NGAL) for timing of initiation of renal replacement therapy for acute kidney injury?
}

\author{
Eric A. Hoste ${ }^{1,2}$, Wim Vandenberghe ${ }^{1}$ \\ ${ }^{1}$ Department of Intensive Care Medicine, Ghent University Hospital, Ghent University, Ghent, Belgium; ${ }^{2}$ Research Foundation-Flanders (FWO), \\ Brussels, Belgium \\ Correspondence to: Eric A. Hoste. ICU, 2K12C, route 1280A, Ghent University Hospital, C. Heymanslaan 10, 9000 Ghent, Belgium. \\ Email: Eric.Hoste@UGent.be. \\ Provenance: This is an invited Editorial commissioned by the Section Editor Xue-Zhong Xing [National Cancer Center (NCC)/Cancer Hospital, \\ Chinese Academy of Medical Sciences (CAMS) and Peking Union Medical College (PUMC), Beijing, China] \\ Comment on: Srisawat N, Laoveeravat P, Limphunudom P, et al. The effect of early renal replacement therapy guided by plasma neutrophil gelatinase \\ associated lipocalin on outcome of acute kidney injury: A feasibility study. J Crit Care 2018;43:36-41.
}

Submitted Aug 27, 2018. Accepted for publication Sep 14, 2018.

doi: $10.21037 /$ jtd.2018.09.110

View this article at: http://dx.doi.org/10.21037/jtd.2018.09.110

Renal replacement therapy (RRT) for acute kidney injury (AKI) has been in use in critically ill patient since the first successful treatments done by William Kolff during the mid-1940-ties (1). Similar to many other invasive treatments used in the intensive care unit (ICU) such as mechanical ventilation, decompressive craniotomy, or intra-aortic balloon pump and despite over 6 decades of experience, the optimal timing of initiation of RRT for AKI is still a matter of debate (2). When there are lifethreatening criteria such as hyperkalemia, pulmonary edema and anuria, or severe acidosis, there is consensus among experts that RRT should be commenced (2-4). Waiting for these criteria to occur may expose the critically ill patient with AKI to the complications of AKI such as fluid overload and accumulation of endogenous uremic substances and nephrotoxins that normally would be removed by the kidneys. One might therefore argue that more early initiation of RRT may mitigate these effects and so lead to better outcomes such as reductions of length of stay, less use of resources, and even improved short-term and longterm survival. There are also clinical data to support this vision. For instance, Zarbock et al. demonstrated recently in the ELAIN study that early initiation of RRT offered a survival benefit on short-term and at 1-year compared to late initiation $(5,6)$. Also, several other prospective and cohort studies suggested we should not wait for these life threatening consequences of AKI to occur, and RRT started more early in the course of AKI offers better outcomes (7-9). However, these findings could not be confirmed by others such as in the recent AKIKI study that compared early initiation of RRT to standard of care (10). Also recent meta-analyses couldn't show a benefit for early initiation of RRT (11-14), although some suggested that there may be a benefit in subgroups such as critically ill patients, surgical ICU patients, or patients treated on continuous RRT (CRRT) $(13,15,16)$.

How can we explain these discrepant findings? An important element in this discussion is the lack of a uniform definition of early initiation of RRT; in ELAIN this was at KDIGO stage 2, while in AKIKI this was KDIGO stage 3 , but many variants have been used in other studies. Also, differences in baseline patient characteristics may explain the differences in study outcomes. For instance, patients in ELAIN were predominantly surgical ICU patients, while AKIKI recruited most medical ICU patients. Obviously, cohort studies are biased by confounding by indication. Finally, the power of the individual studies is too small to draw meaningful conclusions (17). In summary, the data on the potential benefit of early initiation of RRT are at present not clear.

An additional tool to help the clinician decide on the optimal timing would therefore certainly be of help. Since 
biomarkers can identify damage to the kidney and rule out functional AKI, risk assessment by biomarkers may be a logical addition in our current set of diagnostic tools. When there is damage to the kidney this patient is indeed at greater risk for need for RRT.

Srisawat et al. used plasma neutrophil gelatinaseassociated lipocalin (NGAL) as a tool for risk stratification of AKI patients in a small Thai multicenter feasibility study ( $\mathrm{n}=70)$ on early or standard timing of initiation of RRT (18). AKI patients who had a plasma NGAL concentration of $400 \mathrm{ng} / \mathrm{mL}$ or greater were randomized into early or standard timing of initiation of RRT, while patients who had lower NGAL concentration received standard therapy. The authors found patients with a plasma NGAL below $400 \mathrm{ng} / \mathrm{mL}$ were less severely ill, had better clinical outcomes, and importantly, none of these received RRT during the study follow up. In other words, clinicians agreed on not using RRT in patients with NGAL below this specific cut off.

Patients randomized to early and standard use of RRT had similar 28 -day mortality ( $50 \%$ vs. $45 \%, \mathrm{P}=0.72$ ). But, while all patients in the early group received RRT, this was in only $40 \%$ of patients in the standard group. In other words, $60 \%$ of patients in the early group would in normal circumstances, outside the study setting, not have been treated with RRT. This is similar to findings by Gaudry et al. in the AKIKI study and Wald et al. in the STARRTAKI, where mortality of the early and delayed RRT groups were also similar, and $49 \%$ respectively $37 \%$ of patients in the delayed group did not receive RRT $(10,19)$. On first sight this could suggest that almost two-thirds of patients were exposed to a potential hazardous treatment, without having a survival benefit. However, a closer look at the data complicates the interpretation. Srisawat $e t a l$. found that in the standard RRT initiation cohort, patients who did not receive RRT had a trend for higher mortality compared to those who had RRT [7/12 (58\%) vs. 2/8 (25\%), $\mathrm{P}=0.20]$. Also, $12 \%$ of patients in STARRT-AKI died before RRT was commenced (19). Finally, in AKIKI, mortality was highest in the subgroup of patients who were in the delayed group and who were treated with RRT (10). These observations may suggest that some patients were treated too late and could have benefited from an early initiation strategy for RRT.

The crucial issue in these critically ill patients is that patients who do not need RRT, should not be exposed to this therapy. How can the clinician identify these patients? Recently, the Acute Disease Quality Initiative (ADQI) introduced the theoretical concept of balancing the demand and capacity framework for RRT, in which the decision for RRT is driven by the balance between the patients demand for renal support and the existing renal capacity (20). Typically, the standard criteria for RRT only capture a fraction of the demand part, and do not take sufficiently into account severity of current illness and baseline characteristics of the patients. As a bedside clinician we typically combine standard criteria for initiation for RRT with our clinical judgement which includes an assessment of the baseline comorbidities of the patient, and the current severity of illness. Unfortunately, this requires clinical expertise and will vary among different clinicians. Srisawat et al. used a stratification strategy by plasma NGAL to overcome this. Patients with plasma NGAL lower than $400 \mathrm{ng} / \mathrm{mL}$ were not treated by RRT and had better outcomes.

So, does this mean we should use NGAL in adjunction to other elements to decide on initiation of RRT? Not yet! Obviously, given the small number of patients included in the study, these findings lack power and can at most only be hypothesis generating. Apart from that, the decision to not use RRT can have been influenced by the NGAL concentration measured. Also, these findings may be specific to the setting in which the study was performed. It may very well be that patient characteristics and processes of care in other countries or parts of the world may result in a lower specificity for NGAL and renal recovery.

Will NGAL be the definitive biomarker for the decision to initiate RRT? Others have found similar findings in different settings, and a recent meta-analysis, including 16 studies and 3,832 patients, showed that plasma NGAL had an area under the receiver operator characteristic curve (AUC-ROC) for predicting use of RRT of 0.787 , which was evaluated by the authors as a fair prediction, but was in the same order of magnitude as less expensive biomarkers as serum creatinine or cystatin C (21). Importantly, the exact cut off is not established. Srisawat found that $60 \%$ of patients with plasma NGAL $>400 \mathrm{ng} / \mathrm{mL}$ who were in the standard group were not treated with RRT. Others found that especially high cut-offs $(>600 \mathrm{ng} / \mathrm{mL})$ had high specificity for non-recovery of kidney function (21). Of note, not every type of NGAL measurement gives the same message. Rewa et al. found that whole blood NGAL had no predictive power for RRT (22), and similar results are reported for serum NGAL and to a lesser extend 


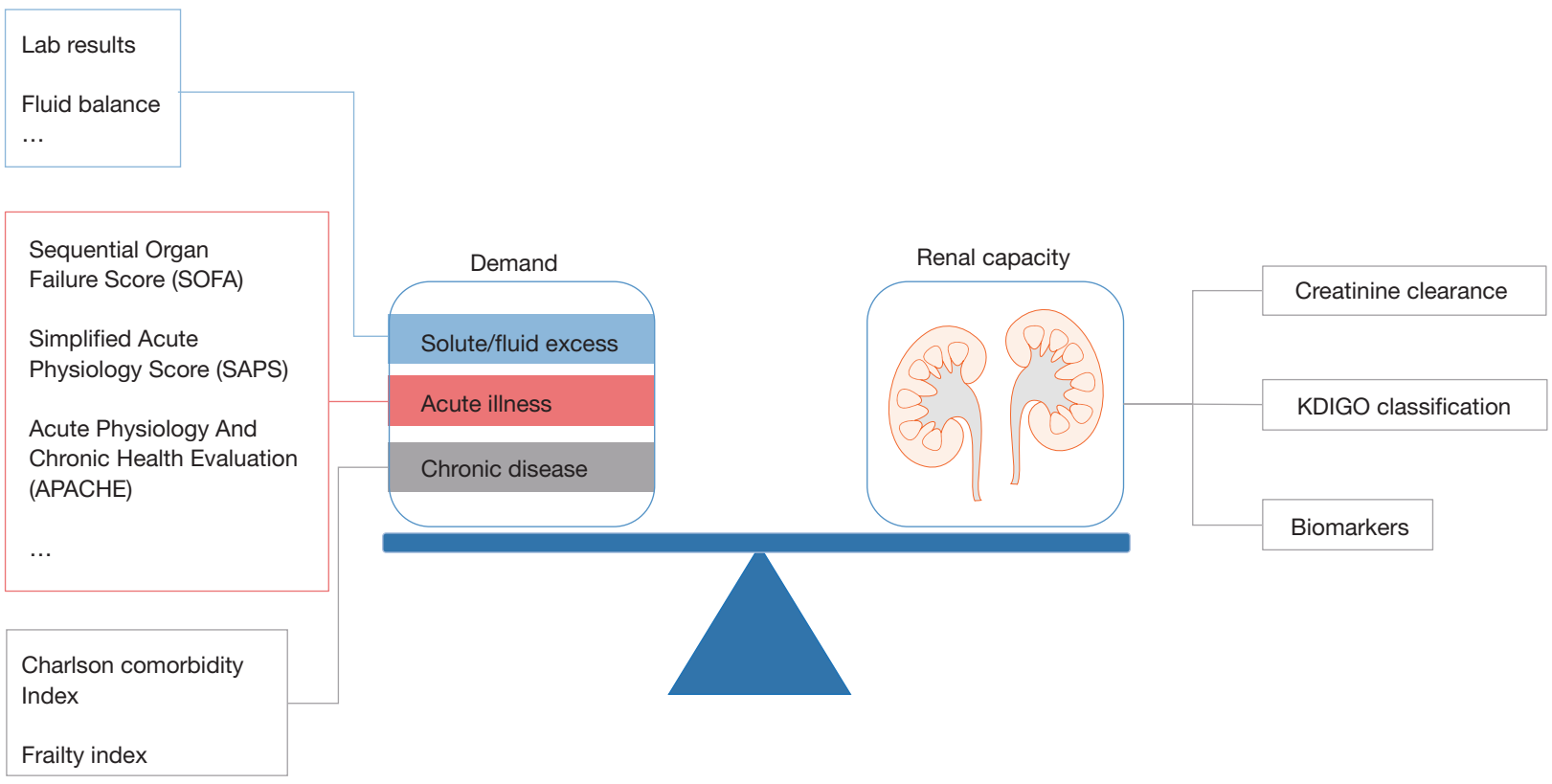

Figure 1 The demand-capacity balance in acute kidney injury patients [modified from Ostermann et al. (20)]. The need for renal replacement therapy is the resultant of the demand and the renal capacity. Demand is the resultant of the chronic disease state (which can be evaluated by scores evaluating chronic disease), the acute disease state (which can be evaluated by acute disease scores), and accumulation of solutes and/or fluid as a consequence of acute kidney injury. The renal capacity can be evaluated by tools evaluating kidney function such as creatinine clearance, KDIGO status and biomarkers for acute kidney injury.

urine NGAL (21). Since plasma NGAL also increases for non-AKI stimuli such as infection and sepsis, conditions often present in critically ill patients with AKI, other more kidney specific biomarkers for AKI may also be of interest. For instance, the urinary biomarkers cystatin C, and urinary TIMP2* IGFBP-7 (the NephroCheck ${ }^{\circledR}$ ) were found to have potential as a marker for use of RRT in the meta-analysis by Klein et al. (21). But also other kidney biomarkers or a panel with a combination of these biomarkers may have potential.

Although, correct identification of patients who do need RRT is probably cost-effective, an important limitation that may limit the use of biomarkers is the cost. At present the STARRT-AKI 2 study is recruiting 2,866 patients to early or standard start of RRT for AKI (NCT02568722). This study excludes patients of whom the treating physicians are convinced they do not need RRT, and patients in whom RRT cannot be delayed. As such they use the clinical expertise of physicians to balance the demand for RRT and the renal capacity. Obviously a much cheaper approach than using plasma NGAL, and mimicking current daily practice. Given the fallacies in prediction of use of RRT and discrepancies between experts, use of a risk score may be a valuable alternative for this clinical expertise. Unfortunately, risk scores are content and region specific, e.g., validated in post cardiac surgery in a specific center, region or country.

In conclusion, increased plasma NGAL may be a valuable screening tool to identify AKI patients at risk for use of RRT. However, the current data do not support widespread use already. Most probably, other biomarkers have similar potential. Since a biomarker adds knowledge on the status of kidney function only, adding knowledge on patients' status with the gut feeling of the treating physician who is helped by a valid risk score would potentially enhance the specificity to identify patients with non-renal recovery (Figure 1). Finally, it is our strong belief that ideal timing of initiation of RRT for AKI will be most likely different in different patient cohorts or different types of AKI.

\section{Acknowledgements}

None. 


\section{Footnote}

Conflicts of Interest: The authors have no conflicts of interest to declare.

\section{References}

1. Kolff WJ. De kunstmatige nier en de ervaringen hiermede opgedaan in de cliniek. Nederlands tijdschrift voor geneeskunde 1946;90:155-8.

2. Bagshaw SM, Darmon M, Ostermann M, et al. Current state of the art for renal replacement therapy in critically ill patients with acute kidney injury. Intensive Care Med 2017;43:841-54.

3. Gibney N, Hoste E, Burdmann EA, et al. Timing of initiation and discontinuation of renal replacement therapy in AKI: unanswered key questions. Clin J Am Soc Nephrol 2008;3:876-80.

4. Vinsonneau C, Allain-Launay E, Blayau C, et al. Renal replacement therapy in adult and pediatric intensive care: Recommendations by an expert panel from the French Intensive Care Society (SRLF) with the French Society of Anesthesia Intensive Care (SFAR) French Group for Pediatric Intensive Care Emergencies (GFRUP) the French Dialysis Society (SFD). Ann Intensive Care 2015;5:58.

5. Zarbock A, Kellum JA, Schmidt C, et al. Effect of Early vs Delayed Initiation of Renal Replacement Therapy on Mortality in Critically Ill Patients With Acute Kidney Injury: The ELAIN Randomized Clinical Trial. JAMA 2016;315:2190-9.

6. Meersch M, Küllmar M, Schmidt C, et al. LongTerm Clinical Outcomes after Early Initiation of RRT in Critically Ill Patients with AKI. J Am Soc Nephrol 2018;29:1011-9.

7. Sugahara S, Suzuki H. Early start on continuous hemodialysis therapy improves survival rate in patients with acute renal failure following coronary bypass surgery. Hemodial Int 2004;8:320-5.

8. Shiao CC, Wu VC, Li WY, et al. Late initiation of renal replacement therapy is associated with worse outcomes in acute kidney injury after major abdominal surgery. Crit Care 2009;13:R171.

9. Chon GR, Chang JW, Huh JW, et al. A comparison of the time from sepsis to inception of continuous renal replacement therapy versus RIFLE criteria in patients with septic acute kidney injury. Shock 2012;38:30-6.

10. Gaudry S, Hajage D, Schortgen F, et al. Initiation
Strategies for Renal-Replacement Therapy in the Intensive Care Unit. N Engl J Med 2016;375:122-33.

11. Bhatt GC, Das RR. Early versus late initiation of renal replacement therapy in patients with acute kidney injury-a systematic review \&amp; metaanalysis of randomized controlled trials. BMC Nephrol 2017;18:78.

12. Feng YM, Yang Y, Han XL, et al. The effect of early versus late initiation of renal replacement therapy in patients with acute kidney injury: A meta-analysis with trial sequential analysis of randomized controlled trials. PLoS One 2017;12:e0174158.

13. Lai TS, Shiao CC, Wang JJ, et al. Earlier versus later initiation of renal replacement therapy among critically ill patients with acute kidney injury: a systematic review and meta-analysis of randomized controlled trials. Ann Intensive Care 2017;7:38.

14. Mavrakanas TA, Aurian-Blajeni DE, Charytan DM. Early versus late initiation of renal replacement therapy in patients with acute kidney injury: a metaanalysis of randomised clinical trials. Swiss Med Wkly 2017;147:w14507.

15. Luo K, Fu S, Fang W, et al. The optimal time of initiation of renal replacement therapy in acute kidney injury: A meta-analysis. Oncotarget 2017;8:68795-808. Erratum in: Correction: The optimal time of initiation of renal replacement therapy in acute kidney injury: A metaanalysis. [Oncotarget. 2018].

16. Wang C, Lv LS, Huang $\mathrm{H}$, et al. Initiation time of renal replacement therapy on patients with acute kidney injury: A systematic review and meta-analysis of 8179 participants. Nephrology (Carlton) 2017;22:7-18.

17. Wald R, Gallagher M, Bagshaw SM. Shedding New Light on an Old Dilemma: Two Trials Examining the Timing of Renal Replacement Therapy Initiation in Acute Kidney Injury. Am J Kidney Dis 2017;69:14-7.

18. Srisawat N, Laoveeravat $\mathrm{P}$, Limphunudom $\mathrm{P}$, et al. The effect of early renal replacement therapy guided by plasma neutrophil gelatinase associated lipocalin on outcome of acute kidney injury: A feasibility study. J Crit Care 2018;43:36-41.

19. Wald R, Adhikari NK, Smith OM, et al. Comparison of standard and accelerated initiation of renal replacement therapy in acute kidney injury. Kidney Int 2015;88:897-904.

20. Ostermann M, Joannidis M, Pani A, et al. Patient Selection and Timing of Continuous Renal Replacement Therapy. Blood Purif 2016;42:224-37. 
21. Klein SJ, Brandtner AK, Lehner GF, et al. Biomarkers for prediction of renal replacement therapy in acute kidney injury: a systematic review and meta-analysis. Intensive Care Med 2018;44:323-36.

Cite this article as: Hoste EA, Vandenberghe W. Plasma neutrophil gelatinase-associated lipocalin (NGAL) for timing of initiation of renal replacement therapy for acute kidney injury? J Thorac Dis 2018;10(Suppl 33):S3989-S3993. doi: 10.21037/ jtd.2018.09.110
22. Rewa O, Wald R, Adhikari NK, et al. Whole-blood neutrophil gelatinase-associated lipocalin to predict adverse events in acute kidney injury: A prospective observational cohort study. J Crit Care 2015;30:1359-64. 